\title{
Vasovagal Reaction
}

National Cancer Institute

\section{Source}

National Cancer Institute. Vasovagal Reaction. NCl Thesaurus. Code C50798.

Sudden drop of the blood pressure, bradycardia, and peripheral vasodilation that may lead to loss of consciousness. It results from an increase in the activity of the vagus nerve. It may be triggered by emotions of fear or pain or gastrointestinal upset and may be relieved by lying down while keeping the legs elevated. 